\title{
Symptoms Experience: A Concept Analysis
}

\author{
Terri S. Armstrong, MS, APRN, BC
}

Purpose/Objectives: To provide a clearly constructed definition of the concept of symptoms experience.

Data Sources: Articles and book chapters.

Data Synthesis: Symptoms experience has not been explored previously. Several approaches to the concept of symptoms have been addressed, including symptom occurrence, symptom distress, and unpleasant symptoms. Limitations of these approaches may include the lack of focus on symptoms as occurring concurrently or in clusters or the multiplicative nature of symptoms. In addition, situational and existential meaning often is not explored.

Conclusions: Symptoms experience is the perception of the frequency, intensity, distress, and meaning of symptoms as they are produced and expressed. Symptoms are multiplicative in nature and may act as catalysts for the occurrence of other symptoms. Antecedents to the symptoms experience include demographic, disease, and individual factors. Consequences include the impact on mood state, psychological status, functional status, quality of life, disease progression, and survival.

Implications for Nursing: Evaluation of symptoms in patients with cancer should include a meaning-centered approach, in which symptoms are evaluated not only for occurrence characteristics and perceived distress but also for the meaning of the symptoms experience to individuals.

$\mathbf{S}$ ymptoms are guideposts for oncology nursing practice. Symptom experience is a concept that often is described in nursing literature as the occurrence of symptoms in clinical and research contexts. Although related terms such as symptom distress have been defined, the experience of symptoms has not been explored fully. Recent work by several researchers has indicated that symptoms often occur in clusters and may be multiplicative in nature (Dodd, Janson, et al., 2001; Lenz, Pugh, Milligan, Gift, \& Suppe, 1997). Therefore, the current analysis will refer to the experience of multiple symptoms as the "symptoms experience."

The process of completing a concept analysis occurs when a concept is systematically and logically investigated to form a clearly constructed definition (Ream \& Richardson, 1996). If a concept is used without first being clearly defined, then any work on which it is based also will be unclear (McKenna, 1997). Unclear theoretical definitions continue to be a major problem in nursing outcomes research (Haberman, 1999; Strickland, 1992). The purpose of this article is to provide an analysis of the concept of symptoms experience using the framework outlined by Walker and Avant (1995). This method includes developing a clear definition, identifying antecedents, defining attributes and consequences of the concept, and developing model and related cases.

\section{Key Points...}

> Current evidence suggests that symptoms may occur in clusters, be multiplicative in nature, and act as catalysts for other symptoms.

> The meaning that the symptoms experience has to patients may influence the symptom occurrence or perceived distress that patients experience.

- Current methods to evaluate symptoms are limited by measuring symptom occurrence and distress individually and often do not consider the meaning or importance of symptoms to patients.

\section{Literature Review}

The word "symptom" can be traced to its Latin origin synthoma and first was used in its present sense in the 1600s (Rhodes \& Watson, 1987). Sign was differentiated from symptom in the 1800 s, with signs being described as alterations that can be ascertained by the sense of the observer and symptoms as changes in the functions of the parts affected (Rhodes \& Watson). Several theories recently have been developed that attempt to explain the occurrence of symptoms and the relation of symptoms to other factors (Lenz et al., 1997; Leventhal \& Johnson, 1983; Rhodes \& Watson). In Leventhal and Johnson's theory of self-regulation, symptoms are concrete representations of disease experienced by individuals as a component of cognitive processing. Their work highlights the differentiation between the occurrence of a symptom (a concrete, objective event) and the emotional response to that event. Lenz et al. developed a middle-range theory of unpleasant symptoms and defined symptoms as "perceived indicators of change in normal functioning as experienced by patients" (p. 3). They conceptualized each symptom to be a multidimensional experience that can be measured separately or in combination with other symptoms. Rhodes and Watson defined symptoms as subjective phenomena regarded by individuals as an indication of a condition departing from normal function, sensation, or appearance or as perceived

Terri S. Armstrong, MS, APRN, BC, is a doctoral student and clinical assistant professor in the School of Nursing at the University of Texas-Houston and a neuro-oncology nurse practitioner for the University of Texas M.D. Anderson Cancer Center in Houston. (Submitted June 2002. Accepted for publication November 22, 2002.)

Digital Object Identifier: 10.1188/03.ONF.601-606 
indicators of change in normal functioning as experienced by patients. Symptoms (including fatigue, nausea, and pain) become known only by the report of the people experiencing them. Common themes of these approaches include the subjective nature of symptoms, the occurrence seen as a departure from normal function, the multidimensional nature, and the inclusion of an emotional response to the symptom. All of the efforts thus far to further describe symptoms indicate that each symptom is a multidimensional experience that can be measured separately or in combination with other symptoms.

The occurrence of symptoms has been delineated further by the concepts that comprise them. Dimensions of each symptom that are common across symptoms and populations are the intensity (strength), timing (duration and frequency), level of distress perceived, and quality (Lenz et al., 1997; Leventhal \& Johnson, 1983; Rhodes \& Watson, 1987). Quality, intensity, and timing often are referred to as characteristics of symptom occurrence, with distress being considered separately (Rhodes \& McDaniel, 1999).

Recently, efforts have focused on the definition and measurement of the distress associated with symptoms. The word "distress" in medieval language was used as a noun indicating distraining or the condition of being distrained (Rhodes \& Watson, 1987). Modern definitions of distress include pressure applied to produce or restrain action, physical or mental suffering, or amount of upset that the sensation causes (Leventhal \& Johnson, 1983; Merriam-Webster, 2001). Symptom distress is defined as the degree or amount of physical or mental upset, anguish, or suffering experienced from a specific symptom (Rhodes \& Watson). McCorkle (1987) furthered work on symptom distress by creating a measurement instrument. She defined measurement as the "systematic attempt to measure the person's level of distress from a specific symptom being experienced" (p. 248). For measuring symptom distress, researchers most often use tools that measure both the occurrence and perceived intensity of symptoms (Haberman, 1999). Most scales measure intensity by having patients rate the severity of a symptom and do not allow for separate measurements of distress. This use of the concept in practice and research highlights incongruency between the definition of distress and what is being measured.

In summary, several approaches related to the description of symptom occurrence and distress have been published in the literature. The approach to the understanding of symptoms has included the concepts of symptoms, symptom occurrence, symptom distress, and unpleasant symptoms. These approaches often take into account the intensity of and distress associated with symptoms but fail to recognize either the meaning of the symptoms to patients, the multiplicative nature of symptoms, or the impact of symptoms on the disease. The concept of "symptoms experience" has not been explored.

\section{Symptoms Experience}

The purpose of this article is to provide a clear definition of symptoms experience, including antecedents, defining attributes, and consequences of symptoms and symptoms experience. The term "experience" first appeared in the 14th century and has been defined as direct observation of, or participation in, events as a basis of knowledge; the conscious events that make up an individual's life; something personally encountered, undergone, or lived through; or the process of directly perceiving events or reality (Merriam-Webster, 2001; Rhodes \& Watson, 1987). The term "symptom experience" first was connected to the concepts of symptom occurrence and symptom distress by Rhodes and Watson in their discussion of nausea and vomiting in terms of the patterns of symptom experience (Rhodes \& McDaniel, 1999). In their development of a model of symptom distress, they defined symptom experience as patients' perceptions and responses to symptom occurrence and distress. However, as Haberman (1999) and McClement, Woodgate, and Degner (1997) have stated, often the occurrence and perceived intensity of symptoms do not correlate with distress. Haberman encouraged researchers to take a meaning-centered approach to assessing the nature of total symptom experience and further defined this experience as a "multidimensional, dynamic process of deriving meaning from a subjective, out-of-the-ordinary sensation-a synthesis of symptom occurrence and perceptions of intensity and distress" (p. 11). Even this definition is lacking, in that it does not take into account the occurrence and potential multiplicative nature of more than one symptom. From this definition, the importance of meaning will be interwoven into the framework currently established for symptoms to analyze the concept of symptoms experience.

For the purpose of the current analysis, symptoms experience is defined as the perception of the frequency, intensity, distress, and meaning occurring as symptoms are produced and expressed. Figure 1 provides a model of the symptoms experience and its components. Various individual factors interact to produce symptoms in individuals. Rhodes and Watson (1987) identified three categories of variables that influence the experience of symptoms - including physiologic, psychological, and situational factors-and saw these components as influencing and being influenced by the symptoms. The perceived components of the symptoms experience include the symptoms' frequency, intensity, distress, and meaning. People's ability to perceive the degree of physical or mental distress is thought to be influenced by various factors, including age, socioeconomic levels, culture, family role, education, health knowledge, values, and past experiences (Rhodes \& McDaniel, 1999; Rhodes \& Watson). This perception of the symptoms experience requires the ability to understand what is obscure. Characteristics of patients (such as individual, disease, and demographic variables) and the impact that the expression of symptoms has for them may modify this understanding. Therefore, this perception may be different based on the individual. The expression or consequences of the symptoms are described as changes in functional and cognitive activities, including adjustment to illness, quality of life, mood, functional status, disease progression, and survival.

\section{Defining Attributes}

Defining attributes are characteristics of the concept that appear repeatedly in the literature (Walker \& Avant, 1995). As noted previously, existing theories and studies of symptoms in various populations have defined key attributes of symptoms as the frequency, intensity, and distress that occur. However, missing from this list of attributes is the meaning of the symptoms and the symptoms experience to patients. According to Rhodes and McDaniel (1999), the meaning that people assign to physical sensations may have profound implications for their physical and psychological health and, therefore, their quality 


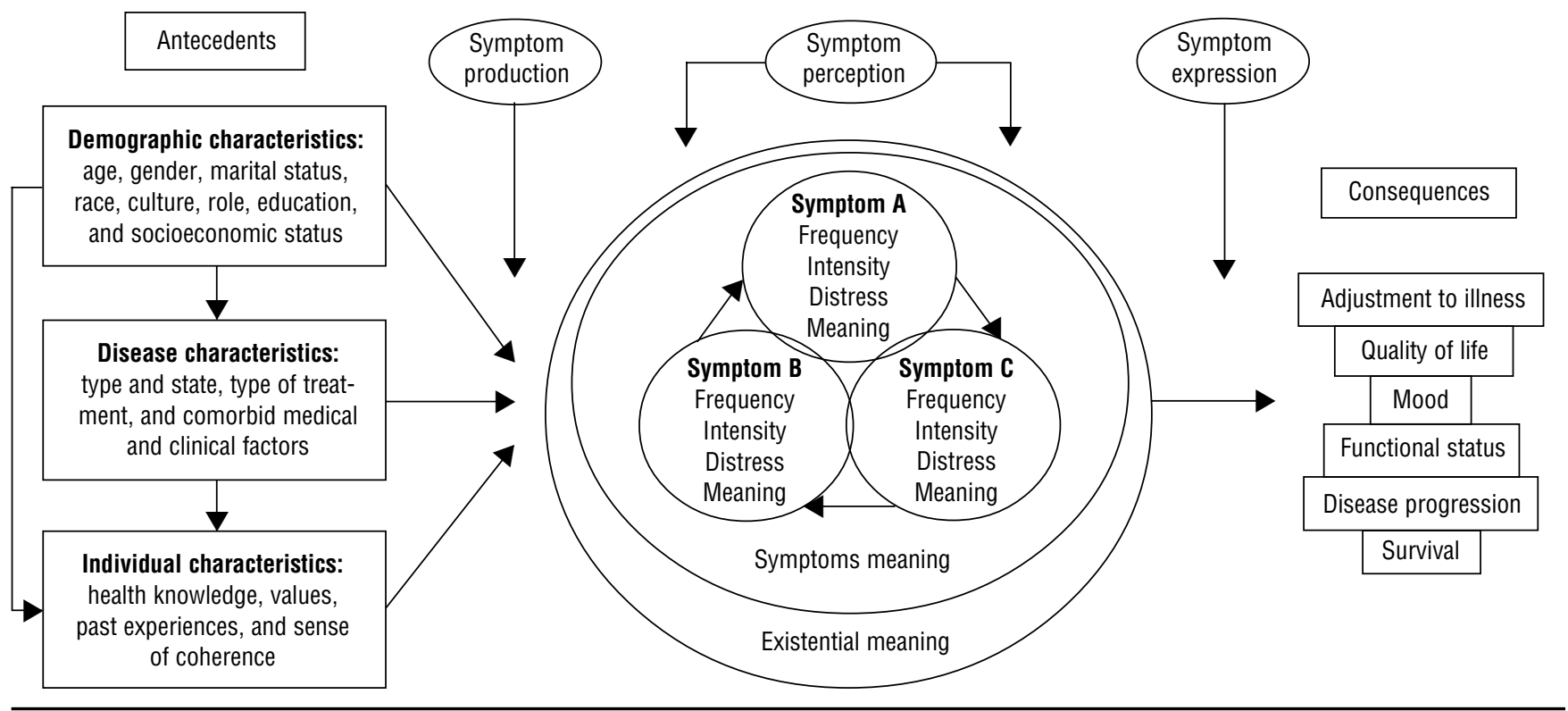

Figure 1. Symptoms Experience Model

of life. Each individual symptom, as well as the interaction of multiple symptoms, has the ability to affect patients' situational meaning (perception of a new event and their capacity to handle it) or existential meaning (global representations of their places in the world) (Richer \& Ezer, 2000). The meaning that patients attribute to the experience of symptoms may influence the perception of a symptom regardless of the frequency or distress associated with the symptom. This meaning may affect the symptoms experience by ascribing a positive or negative perspective on the experience. How patients perceive the impact of these symptoms on daily life is situational meaning. Situational meaning may be triggered by an inability to drive as a result of seizures or to attend a church event as a result of fatigue. Existential meaning may include patients' sense of vulnerability and mortality as a result of symptoms reminding them of their cancer diagnosis. Or the symptoms may have a positive meaning to patients if, for example, they assign symptoms as signifying that the "treatment must be working if I feel this sick" or "the experience has brought my family closer together and allowed us to see the good in each other." For each individual, the symptom considered most distressing may not be the most meaningful symptom to that person. Therefore, the concept of symptoms experience includes the attributes of symptom occurrence and distress, as well as the situational and existential meaning of individual symptoms and the combined impact of multiple symptoms.

Symptoms seldom occur in isolation. Published studies have indicated that patients with solid tumors reported an average of 11-13 symptoms that occurred concurrently and may have influenced the occurrence of other symptoms (Chang, Hwang, Feuerman, \& Kasimis, 2000; Portenoy et al., 1994). According to Lenz et al. (1997), the occurrence of multiple symptoms is likely to result in an experience that is multiplicative rather than additive. Other researchers also have supported this idea (Graydon, Ross, \& Webster, 1995;
Tishelman, Taube, \& Sachs, 1991). Symptoms may occur in clusters (Dodd, Miaskowski, \& Paul, 2001; Sarna, 1998) or act as catalysts for other symptoms or the distress associated with them (Graydon et al.). For example, pain has been reported to be disproportionately more severe when fatigue and nausea are experienced at the same time (DeVito, 1990). Therefore, in understanding the symptoms experience, nurses must view symptoms as influencing and being influenced by the occurrence attributes of other symptoms.

\section{Antecedents}

According to Walker and Avant (1995), identifying antecedents and consequences helps to clarify the attributes and context in which the concept is found. Antecedents affecting the experience of symptoms include demographic characteristics such as gender (Degner \& Sloan, 1995), age (Cimprich, 1999; Collins et al., 2000; Degner \& Sloan), marital status, ethnicity (Bates \& Edwards, 1992; Carter, 1974; Todd, Samaroo, \& Hoffman, 1993), culture (Berg, 1999; Berg \& Taylor, 1999; Cohen \& Palos, 2001; Dodd, Janson, et al., 2001), role (Berg), education (Sarna, 1998), disease characteristics such as type and stage (Cimprich \& Ronis, 2001; Collins et al.; McCorkle \& Quint-Benoliel, 1983), type of treatment (Kaasa, Mastekaasa, $\&$ Thorud, 1988), type of care providers (McCorkle et al., 1994), comorbid medical and clinical factors (Collins et al.; Dales, Spitzer, Schechter, \& Suissa, 1989; Leidy, 1990; Pugh, 1990; Pugh \& Milligan, 1995; Sarna), and individual factors, such as health knowledge, values, and past experiences (Rhodes \& Watson, 1987; Rittenhouse \& Lee, 1993). For the purpose of this analysis, the antecedents to the production of the symptoms experience in patients with cancer have been broadly classified as demographic characteristics (age, gender, marital status, race, culture, role, education, and socioeconomic status), disease characteristics (type and stage of cancer, type and avail- 
ability of treatment, and comorbid medical and clinical factors), and individual characteristics (health knowledge, values, past experiences, and sense of coherence).

\section{Consequences}

Symptoms have been found to affect the production of a variety of health outcomes in various populations. These consequences of the expression of symptoms include patients' adjustment to the illness (Hoskins et al., 1996), quality of life (Cella \& Cherin, 1988; Cimprich, 1999), functional status (Dodd, Miaskowski, et al., 2001; Graydon et al., 1995), psychological state (Cimprich, 1999; McCorkle \& Quint-Benoliel, 1983; Molassiotis et al., 1996; Nerenz, Leventhal, \& Love, 1982), disease progression (Degner \& Sloan, 1995), and survival (Degner \& Sloan; Kaasa et al., 1988; Tamburini, Brunelli, Rosso, \& Ventrafridda, 1996). The occurrence of symptoms can influence functional health status. Patients with several disease states other than cancer and multiple or more severe symptoms have demonstrated a lower functional health status, lower cognitive functioning, less effective role performance, and lower physical performance capabilities (Fawcett, Tulman, \& Myers, 1988; Graydon et al., 1995; Milligan, Parks, \& Lenz, 1991; Pugh \& Milligan, 1995). In patients with cancer, symptom-related distress has been found to significantly and independently predict changes in patient functioning (Given, Given, Azzouz, \& Stommel, 2001; Sarna, 1998). Multiple symptoms in patients with cancer also have been shown to reduce wound healing and lead to immune suppression and progression of metastatic disease (Ben-Eliyahu, Page, \& Shakar, 1999; Kiecolt-Glaser, Page, Marucha, MacCallum, \& Glaser, 1998; Page \& Ben-Eliyahu, 1997). Recently, the occurrence of multiple symptoms in patients with prostate cancer was shown to predict treatment failures and poor therapeutic outcomes (Clark $\&$ Talcott, 2001). For the purpose of the current analysis, the consequences of symptoms expression include patients' adjustment to illness, quality of life, mood, functional status, disease progression, and, ultimately, survival.

\section{Model Case}

A model case is a "real-life" example of the use of the concept. The model case should include all of the critical attributes and no attributes of any other concept.

J.R., a 26-year-old man with a right temporal lobe glioblastoma, described his seizure: "I had that chemical smell again, so I thought, 'here I go again,' sat down on the floor, and tried to stay calm. When I woke up, Luanne was still reading the paper. 'How long did it last?' I asked her. 'About two minutes.' 'Not bad,' I said. 'That's the first one in a week! I knew I would have one; I was so tired from my chemotherapy treatment yesterday.",

S.D., a 64-year-old woman with a glioblastoma of the right temporal lobe, had not had a seizure in a year. While shopping with her daughter, she first smelled a chemical odor and then had a generalized seizure that lasted about two minutes. She woke up in an ambulance on the way to the hospital. "Oh God, I know the tumor is back, how can I handle this? Now I can't drive; how can I go to work?" S.D. was sobbing uncontrollably when she arrived at the emergency room. One week later, she remained weak on her right side, had not returned to work, and was refusing to participate in an upcoming family reunion.

Although both patients had the same diagnosis and experienced identical seizures, the perceived experience of each seizure was very different. Both patients' seizures were influenced by their demographic, disease, and individual factors, leading to a difference in the meaning of the symptom to the person. J.R.'s example highlights the multiplicative nature when he stated that the fatigue from treatment often led to a seizure. J.R. actually experienced seizures frequently, but this did not compound the impact on the meaning of the symptom to him and actually may have diminished the significance of the seizure when compared to S.D. The consequences of the symptom are influenced not only by the occurrence and distress characteristics but also the meaning of the symptom to the person. This is evident in the difference in the perceived meaning and impact on life events between the first and second examples.

\section{Other Cases}

In addition to the development of the model case, Walker and Avant (1995) recommended developing other cases (borderline, contrary, and related cases) to assist in demonstrating the characteristics that make the concept under consideration unique.

Borderline cases are examples or instances that contain some, but not all, of the critical attributes of the concept being examined (Walker \& Avant, 1995). The following is an example.

Jane was caring for Michael, who was hospitalized for seizures related to his brain tumor. Her note stated that Michael had one focal seizure in the past 24 hours. It lasted two minutes and consisted of jerking of his right forearm. This describes the frequency and intensity but does not describe the distress or meaning of the seizure to Michael.

Contrary cases are those that clearly are "not the concept." An example would be Susie spent the day lounging on the beach reading a novel. "Oh," she said, "this is a perfect day. I do not have a care in the world." This example includes none of the characteristics evident in the concept of symptoms experience.

Related cases are instances of concepts that are related to the concept being studied but do not contain all of the critical attributes (Walker \& Avant, 1995). An example would be "Mary sat in her room crying. She had been up vomiting all night and expressed how angry she was at being so sick." This is an example of symptom distress. It describes the person's emotional response to the symptom but does not include the meaning of the symptom.

\section{Conclusions and Implications for Nursing and Research}

Several conceptual models and theories attempt to describe and quantify the impact of symptoms in patients. These models include symptom distress, symptom management, and unpleasant symptoms. The difficulty with these approaches is that they often are unidimensional, measuring only distress and not the impact, importance, or meaning to patients. The symptom distress model, which was used to develop a symptom distress scale, does not consider the multiplicative nature of symptoms. Although the model and the measurement instrument evaluate 
multiple symptoms at one time, distress is measured individually and does not take into account the potential for symptoms to occur in clusters or the overall meaning of the symptoms in patients' lives. Finally, the descriptors for the concepts of symptom distress, unpleasant symptoms, and symptom management are overlapping, leading to confusion about terminology and what concept is the central focus of the study.

Measurement tools that currently exist are designed to measure symptom distress. These tools purport to measure distress but actually measure intensity of symptoms in regard to frequency and severity. Only the University of Texas M.D. Anderson Symptom Distress Scale also includes measures of "interference" of symptoms with activities of daily living (Cleeland et al., 2000). Despite an extensive search, no tool could be found that also allowed patients to rate symptoms according to meaning or importance, independent of distress.

The concept of symptoms experience is an outgrowth of the work on symptom occurrence and symptom distress and allows for the meaning of the symptoms to be considered as well. Focusing on the experience of symptoms, including antecedents and consequences, and adding the value of the meaning of the experience to patients provide an additional layer of complexity but take into account the individual nature of the symptoms experience. Exploring the concept of the experience of symp- toms will provide a basis for further evaluation and clarification of the meaning of symptoms to patients who experience them. In addition, it will provide a framework for further evaluation of factors that previously have been evaluated independently for the relationship to the occurrence of distress of symptoms. Ultimately, understanding the concept of symptoms experience will lend itself to guide assessment techniques and interventions designed to affect symptom occurrence and distress. Factors delineated to be antecedent to the occurrence of symptoms can be targeted for further research to enhance understanding of the impact of these factors on symptoms. In addition, interventions can be explored that alter these factors and have the potential to affect symptom occurrence. This impact will occur by individualizing the plan to the symptoms found to be important to each patient and providing a holistic approach, taking into account disease, individual, and demographic variables that may affect symptoms experienced.

Special thanks to Marlene Cohen, RN, PhD, FAAN, for her support, and Patricia Liehr, RN, PhD, and Geri Wood, RN, PhD, FAAN, for their thoughtful review of this manuscript.

Author Contact: Terri S. Armstrong, MS, APRN, BC, can be reached at tsarmstr@mdanderson.org, with copy to editor at rose_ mary@earthlink.net.

\section{References}

Bates, M.A., \& Edwards, W.T. (1992). Ethnic variations in the chronic pain experience. Ethnic Diseases, 2, 63-83.

Ben-Eliyahu, S., Page, G., \& Shakar, G. (1999). Evidence that stress and surgical intervention promote tumor development by suppressing natural killer cell activity. International Journal of Cancer, 80, 880-888.

Berg, J. (1999). The perimenopausal transition of Filipino American midlife women: Biopsychosocial and cultural dimensions. Nursing Research, 48, $71-77$.

Berg, J., \& Taylor, D. (1999). The symptom experience of Filipino American midlife women. Menopause, 6, 105-114.

Carter, J.J. (1974). Recognizing psychiatric symptoms in black Americans. Geriatrics, 29, 95-99.

Cella, D.F., \& Cherin, E.A. (1988). Quality of life during and after cancer treatment. Comprehensive Therapy, 14(5), 69-75.

Chang, V.T., Hwang, S.S., Feuerman, M., \& Kasimis, B.S. (2000). Symptom and quality of life survey of medical oncology patients at a veteran affairs medical center: A role for symptom assessment. Cancer, 88, 1175-1183.

Cimprich, B. (1999). Pretreatment symptom distress in women newly diagnosed with breast cancer. Cancer Nursing, 22, 185-195.

Cimprich, B., \& Ronis, D.L. (2001). Attention and symptom distress in women with and without breast cancer. Nursing Research, 50(2), 86-94.

Clark, J.A., \& Talcott, J.A. (2001). Symptom indexes to assess outcomes of treatment for early prostate cancer. Medical Care, 39, 1118-1130.

Cleeland, C.S., Mendoza, T.R., Wang, X.S., Chou, C., Harle, M.T., Morrissey, M., et al. (2000). Assessing symptom distress in cancer patients: The M.D. Anderson Symptom Inventory. Cancer, 89, 1634-1646.

Cohen, M.Z., \& Palos, G. (2001). Culturally competent care. Seminars in Oncology Nursing, 17, 153-158.

Collins, J.J., Byrnes, M.E., Dunkel, I.J., Lapin, J., Nadel, T., Thaler, H.T., et al. (2000). The measurement of symptoms in childern with cancer. Journal of Pain and Symptom Management, 19, 363-377.

Dales, R.E., Spitzer, W.O., Schechter, M.T., \& Suissa, S. (1989). The influence of psychological status on respiratory symptom reporting. American Review of Respiratory Diseases, 137, 1459-1463.

Degner, L.F., \& Sloan, J.A. (1995). Symptom distress in newly diagnosed ambulatory cancer patients and as a predictor of survival in lung cancer. Journal of Pain and Symptom Management, 10, 423-431.

DeVito, A.J. (1990). Dyspnea during hospitalizations for acute phase of illness as recalled by patients with chronic obstructive pulmonary disease.
Heart and Lung, 19, 186-191.

Dodd, M., Janson, S., Facione, N., Faucett, J., Froelicher, E.S., Humphreys, J., et al. (2001). Advancing the science of symptom management. Journal of Advanced Nursing, 33, 668-676.

Dodd, M.J., Miaskowski, C., \& Paul, S.M. (2001). Symptom clusters and their effects on the functional status of patients with cancer. Oncology Nursing Forum, 28, 465-470.

Fawcett, J., Tulman, L., \& Myers, S. (1988). Development of the inventory of functional status after child birth. Journal of Nurse-Midwifery, 33, 252-260.

Given, B., Given, C., Azzouz, F., \& Stommel, M. (2001). Physical functioning of elderly cancer patients prior to diagnosis and following initial treatment. Nursing Research, 50, 222-232.

Graydon, J.E., Ross, E., \& Webster, P.M. (1995). Predictors of functioning of patients with chronic obstructive pulmonary disease. Heart and Lung, 24, 369-375.

Haberman, M. (1999). The measurement of symptom distress. In C.H. Yarbro, M.H. Frogge, \& M. Goodman (Eds.), Cancer symptom management. Boston: Jones \& Barlett.

Hoskins, C.N., Baker, S., Sherman, D., Bohlander, J., Bookbinder, M., Budin, W., et al. (1996). Social support and patterns of adjustment to breast cancer. Scholarly Inquiry for Nursing Practice, 10, 99-133.

Kaasa, S., Mastekaasa, A., \& Thorud, E. (1988). Toxicity, physical function and everyday activity reported by patients with inoperable non-small cell lung cancer in a redomized trial (chemotherapy versus radiotherapy). Acta Oncologica, 27, 343-349.

Kiecolt-Glaser, J., Page, G., Marucha, P., MacCallum, R., \& Glaser, R. (1998). Psychological influences on surgical recovery. American Psychologist, 53, 1209-1218.

Leidy, N.K. (1990). A structural model of stress, psychosocial resources, and symptomatic experience in chronic phsyical illness. Nursing Research, 39, 230-236.

Lenz, E.R., Pugh, L.C., Milligan, R.A., Gift, A., \& Suppe, F. (1997). The middle-range theory of unpleasant symptoms: An update. Advances in Nursing Science, 19(3), 14-27.

Leventhal, H., \& Johnson, F.E. (1983). Laboratory and field experimentation development of a theory of self regulation. In P.H. Woolridge, M.H. Schmitt, \& J.K. Skipper (Eds.), Behavior science and nursing theory (pp. 189-262). St. Louis, MO: Mosby.

McClement, S.E., Woodgate, R.L., \& Degner, L. (1997). Symptom distress 
in adult patients with cancer. Cancer Nursing, 20, 236-243.

McCorkle, M., \& Quint-Benoliel, J. (1983). Symptom distress, current concerns and mood disturbance after diagnosis of life-threatening disease. Social Science Medicine, 17, 431-438.

McCorkle, R. (1987). The measurement of symptom distress. Seminars in Oncology Nursing, 3, 248-256.

McCorkle, R., Jepson, C., Malone, D., Lusk, E., Braitman, L., BuhlerWilkerson, K., et al. (1994). The impact of posthospital care on patients with cancer. Research in Nursing and Health, 17, 243-251.

McKenna, H. (1997). Nursing theories and models. London: Rutledge.

Merriam-Webster. (2001). Retrieved October 20, 2001, from http://www .m-w.com/dictionary.htm

Milligan, R.A., Parks, P.L., \& Lenz, E.R. (1991, June). Interaction between social support and predictors of postpartum fatigue. Paper presented at the Eighth Annual Convention of NAACOG, the Organization of Obstetric, Gynecologic, and Neonatal Nurses; Orlando, FL.

Molassiotis, A., Van Den Akker, O.B., Milligan, D.W., Goldman, J.M., Boughton, B.J., \& Holmes, J.A. (1996). Psychological adaptation and symptom distress in bone marrow transplant recipients. Psycho-Oncology, 5, 9-22.

Nerenz, D.R., Leventhal, H., \& Love, R.R. (1982). Factors contributing to emotional distress during cancer chemotherapy. Cancer, 50, 1020-1027.

Page, G., \& Ben-Eliyahu, S. (1997). The immune-suppressive nature of pain. Seminars in Oncology Nursing, 13, 10-15.

Portenoy, R.K., Thaler, H.T., Kornblith, A.B., Lepore, J.M., Friedlander-Klar, K., Coyle, N., et al. (1994). Symptom prevalence, characteristics, and distress in a cancer population. Quality of Life Research, 3, 183-189.

Pugh, L.C. (1990). Psychophysiologic correlates of fatigue during childbearing. Dissertation Abstracts International, 51, $01 \mathrm{~B}$.

Pugh, L.C., \& Milligan, R.A. (1995). Patterns of fatigue during pregnancy. Applied Nursing Research, 8, 140-143.

Ream, E., \& Richardson, A. (1996). Fatigue: A concept analysis. International Journal of Nursing Studies, 33, 519-529.

Rhodes, V.A., \& McDaniel, R.W. (1999). The symptom experience and its impact on quality of life. In C.H. Yarbro, M.H. Frogge, \& M. Goodman
(Eds.), Cancer symptom management (pp. 1-9). Boston: Jones and Barlett.

Rhodes, V.A., \& Watson, P.M. (1987). Symptom distress: The concept past and present. Seminars in Oncology Nursing, 3, 242-247.

Richer, M.C., \& Ezer, H. (2000). Understanding beliefs and meanings in the experience of cancer: A concept analysis. Journal of Advanced Nurisng, 32, $1108-1115$.

Rittenhouse, C., \& Lee, K. (1993). Biopsychosocial correlates of perceived perimenstrual weight gain. Journal of Women's Health, 2, 145-148.

Sarna, L. (1998). Effectiveness of structured nursing assessment of symptom distress in advanced lung cancer. Oncology Nursing Forum, 25, 1041-1048.

Strickland, O.L. (1992). Measures and instruments. In Patient outcomes research: Examining the effectiveness of nursing practice (pp. 145-153). U.S. Department of Health and Human Services. NIH Publication No. 933411. Washington, DC: U.S. Government Printing Office.

Tamburini, M., Brunelli, C., Rosso, S., \& Ventrafridda, V. (1996). Prognostic value of quality of life scores in terminal cancer patients. Journal of Pain and Symptom Management, 11, 32-41.

Tishelman, C., Taube, A., \& Sachs, L. (1991). Self-reported symptom distress in cancer patients: Reflections of disease, illness, or sickness? Social Science Medicine, 33, 1229-1240.

Todd, K.H., Samaroo, N., \& Hoffman, J.R. (1993). Ethnicity as a risk factor for inadequate emergency department analgesia. JAMA, 269, 1537-1539.

Walker, L.O., \& Avant, K.C. (1995). Strategies for theory construction in nursing. Norwalk, CT: Apleton and Lange.

Qis

\section{For more information ...}

$>$ CancerSymptoms.org: Ways to Manage Your Cancer Symptoms www.cancersymptoms.org

Link can be found using ONS Online at www.ons.org.

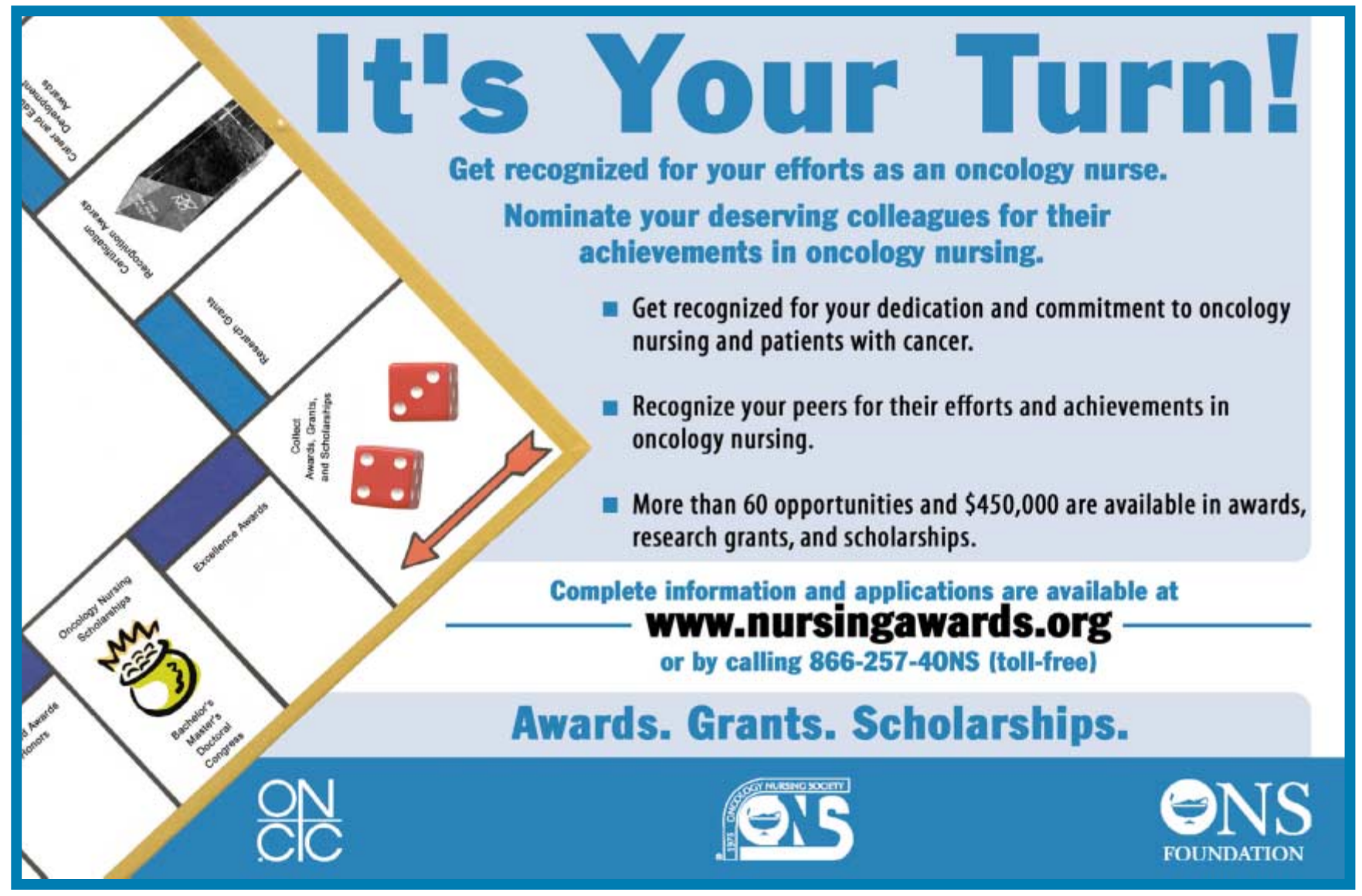


Copyright $\odot 2003$ EBSCO Publishing 